\title{
Scoping review of the literature on shoulder impairments and disability after neck dissection
}

\author{
David P. Goldstein, ${ }^{1 \star}$ Jolie Ringash, ${ }^{2}$ Eric Bissada, ${ }^{1}$ Yves Jaquet, ${ }^{1}$ Jonathan Irish, ${ }^{1}$ Douglas Chepeha,${ }^{3}$ Aileen M. Davis ${ }^{4}$ \\ ${ }^{1}$ Department of Surgical Oncology, Princess Margaret Hospital, Department of Otolaryngology - Head and Neck Surgery, University of Toronto, Toronto, Ontario, Canada, \\ ${ }^{2}$ Department of Radiation Oncology, Princess Margaret Hospital, University of Toronto, Toronto, Ontario, Canada, ${ }^{3}$ Department of Otolaryngology - Head and Neck Surgery, \\ University of Michigan, Ann Arbor, Michigan, ${ }^{4}$ Division of Health Care and Outcomes Research, Toronto Western Research Institute, University Health Network, University of \\ Toronto, Toronto, Ontario, Canada.
}

Accepted 6 December 2012

Published online 1 April 2013 in Wiley Online Library (wileyonlinelibrary.com). D0l 10.1002/hed.23243

ABSTRACT: Background. The purpose of this article was to provide a review of the literature on shoulder disability after neck dissection.

Methods. A literature review was performed using Ovid Medline and Embase databases. A total of 306 abstracts and 78 full-text articles were reviewed. Forty-two articles were eligible for inclusion.

Results. Patients undergoing nerve-sacrifice neck dissections have greater disability and lower quality of life scores than those undergoing neck dissections with the least manipulation (ie, selective neck dissections). Shoulder impairments can still occur in patients undergoing selective neck dissections. Disability typically improves over time in patients undergoing nerve-sparing neck dissections.

Conclusion. There was significant variability in the literature in terms of the prevalence and recovery of shoulder morbidity after neck dissection. This variability may not just be related to surgical technique or rehabilitation, but also to study design, definitions, and the variability in disability questionnaires used. (c) 2013 Wiley Periodicals, Inc. Head Neck 36: 299-308, 2014

KEY WORDS: shoulder disability, neck dissection, literature review, head and neck cancer

\section{INTRODUCTION}

Health status outcome measurement such as disability and quality of life (QOL) has become a critical issue in both research and clinical endeavors in head and neck oncology. ${ }^{1}$ One of the most widely accepted definitions of disability is the World Health Organization (WHO) International Classification of Functioning (ICF), Disability and Health taxonomy that identifies the consequences of disease and treatment; namely, impairments, activity limitations, and restrictions in social participation. ${ }^{2-4}$ Impairment is defined by the ICF as a significant deviation or loss in the physiologic function(s) of a body system or an anatomic part of the body. ${ }^{2,4}$ Activity limitations are difficulties an individual may face in the execution of a task or action, whereas participation restrictions are problems an individual may experience in involvement in a life situation. ${ }^{2,4}$ In accordance with the ICF, a complete assessment of outcome for any health condition or intervention requires an evaluation of health status outcomes in these domains. ${ }^{2,4}$ Health-related quality of life (QOL) is a sub-

*Corresponding author: D. P. Goldstein, Princess Margaret Hospital, Rm 3-952, 610 University Avenue, Toronto, Ontario, M5G 2M9, Canada.

E-mail: david.goldstein@uhn.on.ca

Contract grant sponsor: This work was supported by a Cancer Care Ontario, Ontario Cancer Research Network, and Canadian Institutes for Health Research grant. jective, multi-attribute construct defined by the WHO as "an individual's perception of their position in life in the context of the culture and value systems in which they live and in relation to their goals, standards, and concerns., 5,6

It has long been recognized that the temporary or permanent denervation of the trapezius muscle secondary to spinal accessory nerve (cranial nerve XI) injury during a neck dissection results in shoulder-related disability. In 1952, Ewing and Martin ${ }^{7}$ were the first to report that resection of cranial nerve XI with a radical neck dissection resulted in significant shoulder impairments, including pain, and reduction in strength and range of motion (ROM). In 1961, Nahum and Marmor reported that shoulder impairment after a radical neck dissection is characterized by shoulder droop, winged scapula, inability to shrug, and a dull nonlocalizing pain that was exacerbated by movement, particularly shoulder abduction. ${ }^{8}$ Other contemporary authors reported that these shoulder impairments resulted in significant limitations in daily activities, work-related tasks, and recreation. ${ }^{9,10}$ With the recognition of the impact of shoulder disability on patients, surgeons developed modifications of the radical neck dissection, including the modified radical neck dissection and selective neck dissections that preserved cranial nerve XI without compromising oncologic outcomes. ${ }^{11,12}$ However, despite preservation of cranial nerve XI, shoulder disability was still reported after these less radical procedures. ${ }^{13,14}$

There has been a significant amount of literature assessing shoulder disability in patients undergoing both nerve- 
sparing and nerve-sacrificing neck dissections. ${ }^{15-18}$ The purpose of this scoping review was to provide a literature review of shoulder impairments, activity limitations, and participation restrictions after neck dissection. The overall goal was to highlight the extent of the problem, as well as the significant variability in outcomes and results, and the wide variability in the measurement tools used to assess these outcomes. By identifying the gaps in the existing literature, areas of focus for future efforts can be illuminated.

\section{METHODS}

\section{Search strategy}

A scoping review of the literature evaluating shoulder impairments, activity limitations, and participation restrictions after neck dissection for head and neck cancer was performed using Ovid Medline and Embase databases (from 1980 to July 2011). The electronic search was restricted to articles published in the English language using the following medical subject heading terms or text words: shoulder, upper extremity, disability, activity limitations, impairment, function, limitations, questionnaire, spinal accessory nerve, shoulder syndrome, morbidity, pain, symptoms, quality of life, neck dissection, and head and neck cancer. The electronic search was supplemented by cross-referencing potentially relevant citations from the reference lists of identified publications. All abstracts from the search strategy were reviewed for eligibility.

\section{Selection of studies for inclusion}

All abstracts and citations were reviewed for relevance. Two independent reviewers reviewed any abstracts or citations deemed potentially relevant in full-text form. Full-text articles were included in this review only if consensus was achieved between reviewers based on the following inclusion and exclusion criteria.

\section{Inclusion and exclusion criteria}

Studies were eligible for inclusion if they reported on either evaluation of patients' impairments, activity limitations and/or participation restrictions, or evaluation of shoulder range of motion, strength, or electrophysiological studies of trapezius function after neck dissection. Studies must have included more than 5 patients. Review articles were excluded. Studies that evaluated methods/ techniques of prevention of shoulder disability after neck dissection were also excluded. The studies that met inclusion criteria were reviewed in terms of the construct being evaluated, study design (ie, cross-sectional vs prospective), outcome measures, and the reported outcomes.

\section{RESULTS}

There were a total of 306 abstracts or citations reviewed and 78 full-text articles reviewed in duplicate for eligibility for inclusion. There were a total of 42 articles eligible for inclusion. For the review, the terms used by the authors to describe the construct measured will be provided in quotations. For the studies described in the following literature review, Tables 1, 2, 3, and 4

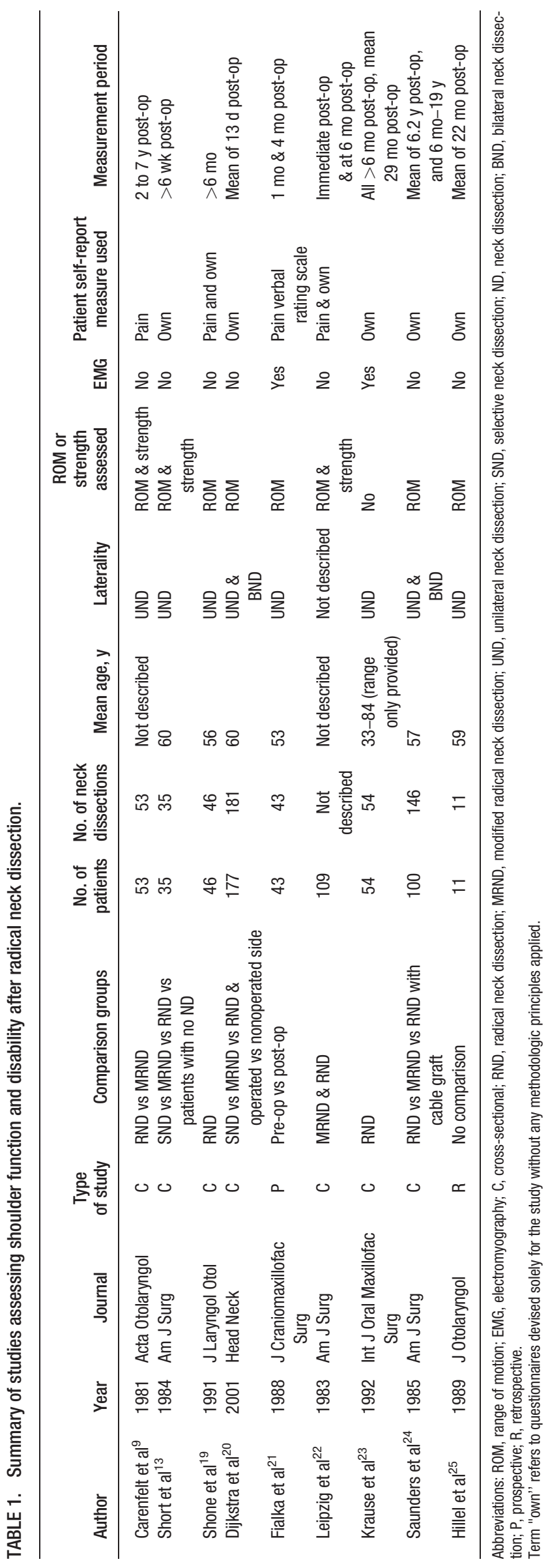




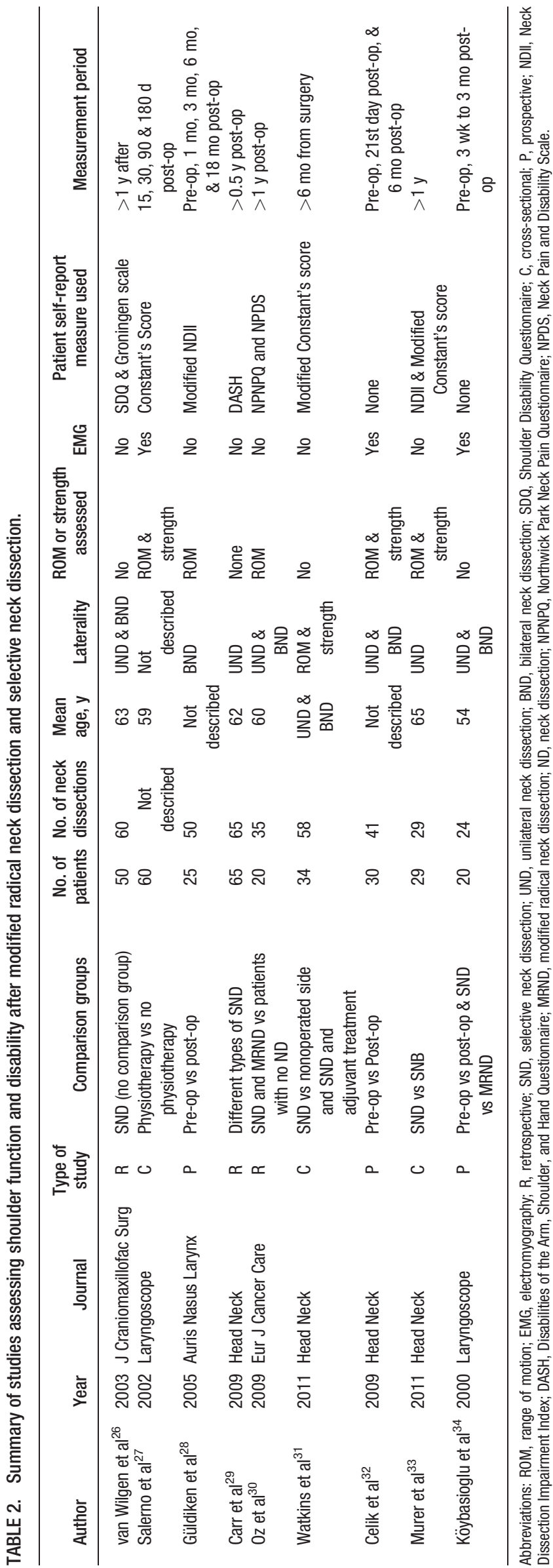

highlight the year of publication, sample size, comparison groups, outcome measures, and time of assessment.

\section{Studies evaluating shoulder-related outcomes after neck dissection}

Radical neck dissections (nerve-sacrifice neck dissections). Shoulder pain is the most frequent "complaint" after radical neck dissection. The degree of severity of pain is highly variable between studies: severe pain requiring daily use of analgesia is unusual. ${ }^{9,19,35,57}$ Dijkstra et $\mathrm{al}^{20}{ }^{20}$ using a visual analog scale, reported that $70 \%$ of 42 patients who underwent radical neck dissection reported having pain before discharge from the hospital. Fialka et al, ${ }^{21}$ using a pain verbal rating scale, found that $77 \%$ of 43 patients who underwent radical neck dissection, assessed between 1 and 6 months after surgery, had severe or strong shoulder pain. Shone et $\mathrm{al}^{19}$ noted that only $30 \%$ of 46 patients who were all greater than 6 months from their unilateral radical neck dissection reported moderate-severe or severe pain related to the shoulder on a questionnaire devised by the authors. An additional $30 \%$ of patients reported some degree of pain most days or every day. Carenfelt et $\mathrm{al}^{9}$ noted that discomfort and pain only became significant beyond 3 months after surgery. Although Short et al ${ }^{13}$ reported that 9 of 12 patients $(75 \%)$ in their series had some degree of shoulder pain, the average score was 2.7 on a pain scale of zero to 5 .

Impairments in shoulder strength and ROM have frequently been described after radical neck dissection. Leipzig et $\mathrm{al}^{22}$ found that nearly all of their 35 patients who underwent a radical neck dissection had reduced shoulder strength and ROM measured at 6 months after surgery, compared with their preoperative assessment. Krause et $\mathrm{al}^{23}$ noted variability in severity in both clinical examination and electromyography (EMG) results in 54 patients who underwent radical neck dissection. With a mean time from surgery of 29 months, they also found that $31 \%$ experienced severe limitations of ROM combined with severe pain, whereas $41 \%$ only mild discomfort. Other authors, including Fialka et $\mathrm{al}^{21}$ and Saunders et $\mathrm{al}^{24}$ have also reported variable results. The most commonly reported activity limitations in daily life included lifting, raising or carrying objects, and leaning or lying on the ipsilateral shoulder. ${ }^{15,25,58}$ Shone et al ${ }^{19}$ found that $77 \%$ of patients had difficulty with everyday tasks such as dressing, combing their hair, hanging up clothes, and reaching a high shelf. Nine patients $(19.5 \%)$ reported that they could no longer pursue activities they enjoyed (ie, participation restrictions) before surgery such as tennis, darts, gardening, or fishing and 11 of 24 patients (46\%) who were employed before surgery stopped working in the long-term (ie, 6 months) specifically because of shoulderrelated problems. Of those patients still employed at the time of the study, 12 of these patients changed their occupation because of their shoulder problems. Table 1 summarizes the studies assessing shoulder function and disability after radical neck dissection in terms of year of publication, sample size, comparison groups, intervention, outcome measures, and time of assessment. 


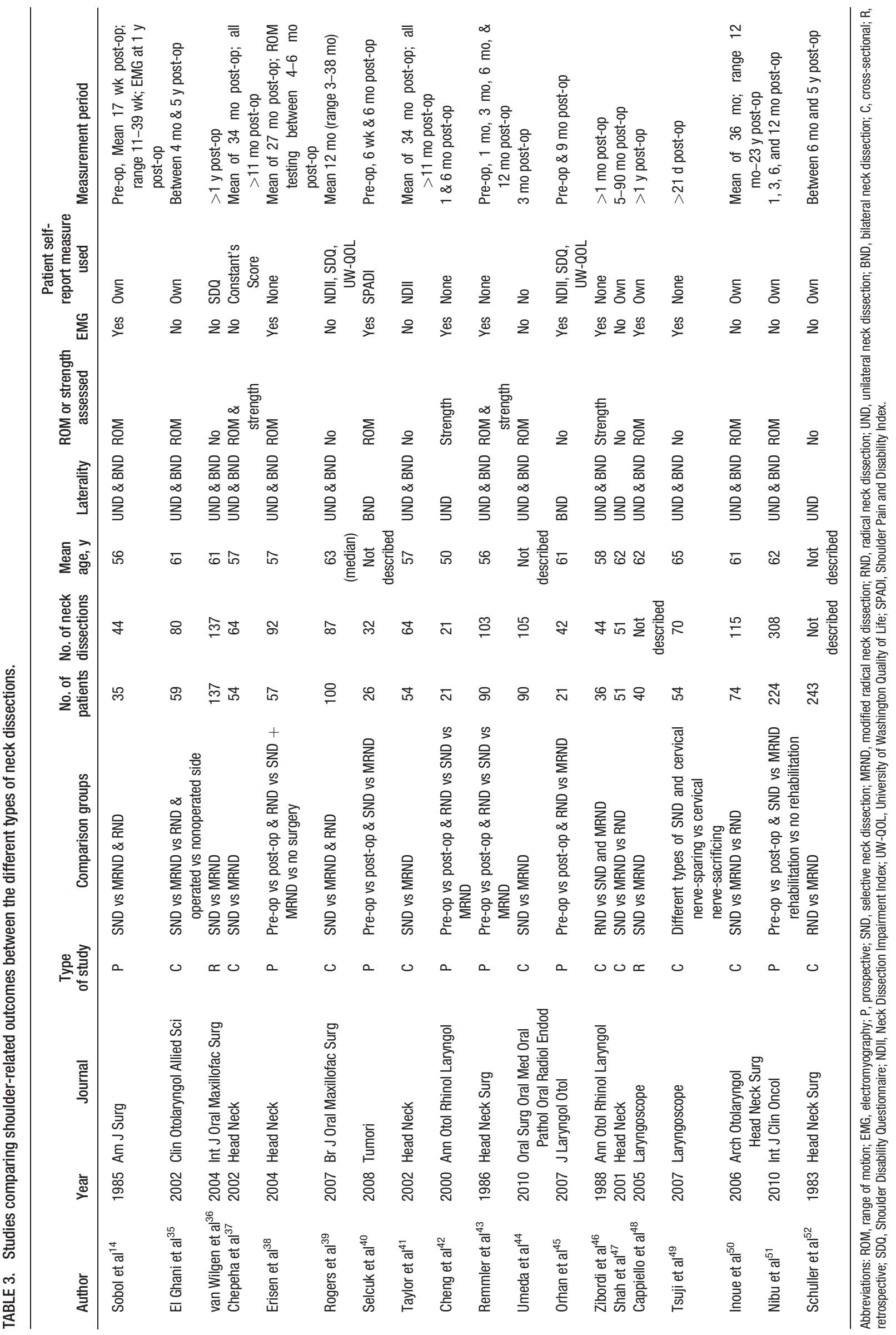




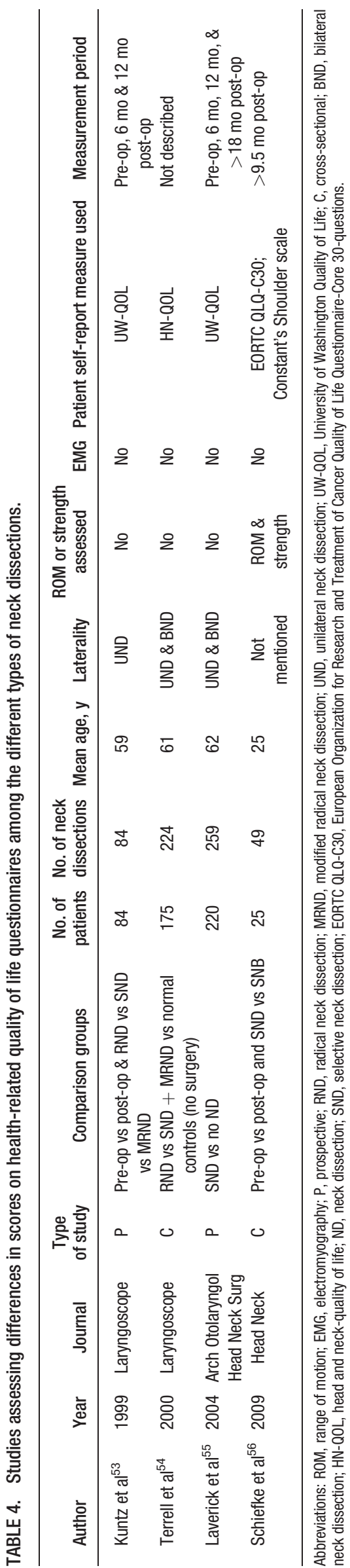

Selective and modified radical neck dissections (nerve-sparing neck dissections). There is significant variability in terms of the reported prevalence of shoulder impairments and activity limitations after modified radical neck dissection and selective neck dissection. ${ }^{20,36}$ Some of the variability can be attributed to how authors defined and measured "disability." Some authors measured symptoms alone, ${ }^{21,26,59}$ others evaluated impairments in ROM and strength, ${ }^{27,37,38}$ whereas other authors reported on activity limitations. ${ }^{39-41,53,60}$ No standard tool was used to assess activity limitations between studies. Table 2 summarizes the studies assessing shoulder function and disability after modified radical neck dissection and selective neck dissection in terms of year of publication, sample size, comparison groups, intervention, outcome measures, and time of assessment.

Salerno et $\mathrm{al}^{27}$ reported that complaints of "shoulder impairment" after modified radical neck dissection occurred in up to $40 \%$ of patients. Van Wilgen et $\mathrm{al}^{26}$ reported that $28 \%$ of 52 patients who underwent selective neck dissection experienced long-term pain, particularly with activities such as moving the arm or shoulder, reaching above the shoulder, or carrying heavy objects. Güldiken et $\mathrm{al}^{28}$ assessed 25 patients before and after "functional neck dissection" (with no clarification of the exact type of nerve-sparing neck dissection) using the Neck Dissection Impairment Index (NDII) questionnaire. The mean NDII score at 1 year after surgery was 98.2 of 100 (range, 95-100), with higher scores signifying less disability. Although minimal disability was noted, pain and stiffness scores at the last follow-up were worse than preoperative scores $(p<.005)$. On ROM assessment, there were no significant differences between the preoperative and postoperative scores at 18 months. Carr et $\mathrm{al}^{29}$ also found, when using the Disabilities of the Arm Shoulder and Hand Questionnaire (DASH), that patients who underwent selective neck dissection had minimal postoperative dysfunction. Overall, 23\% reported no upper limb dysfunction, 54\% reported mild upper limb dysfunction, $1 \%$ reported moderate dysfunction, and $8 \%$ reported severe dysfunction.

Although associated with minimal shoulder disability, selective neck dissection is not without its morbidity. $\mathrm{Oz}$ and $\mathrm{Memis}^{30}$ compared shoulder and neck pain, along with ROM, between 20 patients who underwent a cranial nerve XI-sparing neck dissection and 20 healthy agematched subjects. Two pain scales were used for assessment; the Northwick Park Pain Questionnaire and the Neck Pain and Disability Scale. On average, patients were 16 months after their neck dissection. Although not severe in magnitude, shoulder pain scores were higher and ROM testing (goniometric evaluation) lower in the surgical group than in the control group. ${ }^{30}$ Murer et $\mathrm{al}^{33}$ evaluated patients undergoing either sentinel node biopsy (with no cranial nerve XI dissection) or elective neck dissection (type not described) with the NDII and Constant Shoulder Score more than 1 year after surgery. Overall mean scores on both instruments were high (ie, minimal morbidity); however, scores were statistically significantly higher (ie, lower disability) in the patients undergoing sentinel node biopsy. Watkins et $\mathrm{al}^{31}$ evaluated shoulder function in 34 patients undergoing selective neck dissection using a modified Constant's Score. Their results 
showed a negative effect on shoulder function after selective neck dissection, in spite of saving cranial nerve XI when compared to the nonoperated side. A few studies have assessed shoulder morbidity after selective neck dissection without manipulation of cranial nerve XI by avoiding dissection of level IIb. Celik et $\mathrm{al}^{32}$ found no statistically significant differences in shoulder movement and strength testing between preoperative values and values at either the $21 \mathrm{st}$ day or the 6-month postoperative follow-up assessment. There was a statistically significant change in EMG scores between preoperative and both postoperative time periods, with the worst scores reported at 21 days postoperative. In a similar patient population, Koybaşioğlu et $\mathrm{al}^{61}$ found that distal latencies and compound muscle action potentials were statistically significantly lower at the third week and third month postoperative compared with preoperative values. Although no motor unit potentials were found in 8 patients in the early postoperative period, in the late postoperative period, there were no motor unit potential losses in any of the patients.

\section{Studies comparing shoulder-related outcomes between the different types of neck dissections}

Shoulder impairment tends to occur in the early postoperative period after most radical neck dissections and a significant proportion of nerve-sparing neck dissections, ${ }^{27,60}$ although patients who underwent selective neck dissection exhibit less impairment and fewer activity limitations. ${ }^{14,20,36,37,40}$ The initial decline in shoulder function tends to be followed by progressive improvement in both patients who undergo selective neck dissection and modified radical neck dissection as reinnervation occurs. ${ }^{22}$ This typically takes between 6 months and 1 year, depending upon the degree of injury. ${ }^{42,43,62}$ Although similar rates of shoulder impairment in the early postoperative period have been reported in patients who underwent radical neck dissection and modified radical neck dissection, the patients who underwent radical neck dissection have significantly worse shoulder ROM, strength, pain, and activity limitations than those who underwent modified radical neck dissection in the longer term (ie, $>6$ months after surgery). ${ }^{13,14,22-24,28,42-44,53,54}$ Table 3 summarizes the studies comparing shoulder-related outcomes between the different types of neck dissections.

Using a questionnaire modified from NDII, Orhan et $\mathrm{al}^{45}$ noted that patients who underwent modified radical neck dissections reported significantly less "disability", compared with patients who underwent radical neck dissection. On electrophysiological assessment, decreases in amplitude and EMG scores were more prominent in the radical neck dissection group compared with the modified radical neck dissection group. The amplitude of the trapezius motor response improved with time in the patients who underwent modified radical neck dissection but patients never reached their preoperative values by 9 months after surgery. Zibordi et $\mathrm{al}^{46}$ compared patients undergoing functional neck dissections (ie, those that included lateral cervical node bearing tissue while preserving the sternocleidomastoid, cranial nerve XI and IJV) with 10 patients who underwent a radical neck dis- section. Mean follow-up time from surgery was 3.5 years (range, 1 month-10 years). Both strength testing and EMG results were significantly better in the functional neck dissection group. In the functional neck dissection group, EMG scores were reported as normal in $84.1 \%$ of patients and there were slight peripheral neurogenic lesions in $13.6 \%$ of patients. In comparison, $100 \%$ of the 10 patients who underwent radical neck dissection had severe peripheral neurogenic lesions. Similar results were found for muscle testing for each group. Umeda et $\mathrm{al}^{44}$ recently reported that there was severe limitation in shoulder abduction at 3 months after all nerve-sacrifice surgeries, whereas 90 of 96 patients who underwent a cranial nerve XI-preserving neck dissection maintained their normal shoulder function. Based on patient interviews, El Ghani et $\mathrm{al}^{35}$ reported that $50 \%$ of patients who underwent a radical neck dissection reported severe "activity disability," compared with less than $26 \%$ of patients who underwent modified radical neck dissection $(p=.009)$. Pain was also significantly worse after a radical neck dissection. Shah et $\mathrm{al}^{47}$ and Saunders et $\mathrm{al}^{24}$ reported similar findings with significantly less "disability" or "shoulderrelated symptoms" after modified radical neck dissection compared with radical neck dissection when measured greater than 6 months from surgery.

There is variability in the literature on whether patients who undergo selective neck dissection have less shoulderrelated impairment, activity limitation, and participation restrictions than patients who undergo modified radical neck dissection. Van Wilgen et $\mathrm{al}^{36}$ evaluated patients using the shoulder disability questionnaire (SDQ) and found that there was greater "disability" after modified radical neck dissection compared with selective neck dissection. Similar results were reported by Taylor et $\mathrm{al}^{41}$ using the NDII and Chepeha et $\mathrm{al}^{37}$ using the Constant Shoulder Score. Both noted that patients who underwent selective neck dissection had statistically significantly higher NDII scores (ie, less disability) than those who underwent modified radical neck dissection. All patients in these 3 studies were at least 11 months from surgery. On the other hand, Cappiello et $\mathrm{al}^{48}$ reported that on subjective testing (greater than 1 year from surgery) there was no difference between patients who underwent a selective neck dissection with or without level $\mathrm{V}$ dissection in terms of self-reported shoulder pain, functional limitations, and shoulder strength. However, level V dissection was also associated with greater EMG abnormalities. In both groups, electroneurographic data showed statistically significant abnormalities on the operated side compared with the nonoperated shoulder. In contrast, Tsuji et al $^{49}$ were not able to show any statistically significant differences on EMG evaluation of the trapezius muscle of patients, based on whether or not dissection of level $\mathrm{V}$ was done, in all patients undergoing nerve-sparing neck dissections. However, the group that had level V dissected had the lowest mean EMG scores (not statistically significant).

Inoue et $\mathrm{al}^{50}$ assessed patients who underwent different types of neck dissections greater than 1 year prior. Scores for stiffness and appearance were lower in patients who underwent any type of neck dissection compared with a control group of patients who did not undergo a neck 
dissection $(p<.001)$. Scores for pain and numbness in patients who underwent selective neck dissection were significantly better than those who underwent modified radical neck dissection or radical neck dissection. Shoulder droop and arm abduction scores in patients after radical neck dissection was significantly worse than in those who underwent either selective neck dissection or modified radical neck dissection; however, all neck dissection patients had reduced arm abduction compared with the control group. Similarly, Nibu et $\mathrm{al}^{51}$ reported that patients who underwent a modified radical neck dissection and radical neck dissection greater than 1 year prior reported more shoulder pain and numbness compared to patients who underwent a selective neck dissection. Furthermore, in those that underwent a selective neck dissection, the shoulder and neck pain tended to improve, whereas no improvement was observed after radical neck dissection or modified radical neck dissection. Scores for arm abduction tests were significantly better in patients in whom the cranial nerve XI was preserved compared to scores in whom it was resected. Rogers et $\mathrm{al}^{39}$ used 3 measures that include assessment of shoulder disability to evaluate patients who underwent selective neck dissection, modified radical neck dissection, or radical neck dissection. The mean time from surgery was 1 year. They found that the highest levels of shoulder disability were reported by patients after radical neck dissection and the lowest after selective neck dissection. Scores were similar between the selective neck dissection patients and those who never underwent a neck dissection. Schuller et $\mathrm{al}^{52}$ found that of 203 patients employed before neck dissection, only 104 (51.2\%) returned to their usual occupation after treatment. A similar rate of unemployment was found between patients who underwent modified radical neck dissection and radical neck dissection.

\section{Studies assessing differences in scores on health related quality of life questionnaires among the different types of neck dissections}

Studies have attempted to assess the relationship between the type of neck dissection and scores on QOL questionnaires (Table 4). Using the University of Washington Quality of Life (UW-QOL), a head and neck cancer-specific QOL questionnaire, Laverick et $\mathrm{al}^{55}$ found that those who underwent unilateral selective neck dissection had lower scores (ie, worse QOL) more than 1 year after surgery compared with patients who did not undergo a neck dissection, but better scores than those who underwent a modified radical neck dissection. Similarly, Schiefke et al, ${ }^{56}$ using the general health-related European Organization for Research and Treatment of Cancer Quality of Life Questionnaire-Core 30-questions, showed a statistically significant decrease in QOL in those having undergone selective neck dissection as opposed to the normal population. However, there was no statistically significant differences in QOL questionnaire scores seen between those having undergone sentinel lymph node biopsy and those undergoing selective neck dissections, despite differences on the Constant Shoulder Score. ${ }^{33}$ Van Wilgen et $\mathrm{al}^{63}$ found that shoulder abduction and pain scores (measured on a visual analog scale) in patients who underwent a neck dissection (radical neck dissection, modified radical neck dissection, or selective neck dissection) were significantly related to several domains (socialfunctioning domain and limitation from physical-problem domain) on a general overall QOL questionnaire (RAND36 QOL). Kuntz et $a^{53}$ showed that although the shoulder domain scores on the UW-QOL questionnaire differed based on type of neck dissection, there were no significant differences in the other QOL domain scores (subjective appearance, activity, recreation, chewing, swallowing, or speech). Rogers et $\mathrm{al}^{39}$ reported that, despite objective and subjective shoulder deficits after neck dissection, patients reported shoulder impairment as significantly less important to their QOL than other functional deficits, such as speech and swallowing difficulties.

\section{DISCUSSION}

Shoulder-related morbidity has been a long-recognized problem after neck dissection. There still remains considerable uncertainty about the true extent of impairments, activity limitations, and participation restrictions, particularly after nerve-sparing neck dissections. There has been a considerable amount of research in this area, as well as some review articles. ${ }^{15-17}$ This current systematic review reports on the scope of the problem of shoulder morbidity in patients undergoing neck dissection. The purpose of the review is not only to report on shoulder-related outcomes but also to highlight the weaknesses of the literature.

As has been noted for many decades, patients who underwent radical neck dissection frequently have shoulder impairments, particularly pain and functional limitations, which can interfere with their ability to function in everyday life. Impairments and activity limitations in these patients tend to be prolonged and moderate to severe. However, there is variability in the reported frequency and severity of pain, as well as the prevalence of activity limitations or participation restrictions. Much of this variability is attributed to the different patterns of innervation to the trapezius, with the cervical plexus contributing significant innervation in up to $25 \%$ of patients as confirmed by EMG studies. 7,21,23,49

There is also significant variability in the reported prevalence of shoulder impairments, activity limitations, and participation restrictions after all types of modified radical neck dissection and selective neck dissection. Overall, it seems that patients with nerve-sparing neck dissections less frequently have long-term shoulder-related morbidity than those that undergo a radical neck dissection. The long-term differences between patients who undergo modified radical neck dissection and selective neck dissection remain less clear, with the literature showing that patients who undergo selective neck dissection tend to have better outcomes. The difference between patients who underwent selective neck dissection and modified radical neck dissection is greater in the early postoperative period and seems to decline with time as recovery of nerve function occurs. In patients undergoing selective neck dissection, many have no reduction in reported impairments, including pain and activity limitations, and if they do have short-term changes, these seem to 
improve in the vast majority of patients. However, in both groups, the frequency and severity of shoulder disability, as well as findings on electrophysiologic studies, varies between studies. Some of this variability in morbidity within and between neck dissection groups may be attributed to the differences in innervation patterns to the trapezius muscle, as well as the development of adhesive capsulitis of the shoulder joint in patients who do not undergo physiotherapy. ${ }^{23,40,64-66}$ Variability in the literature can also be attributed to the inherent weaknesses of the studies and, in particular, the multiplicity of constructs measured and the variability of the measures that have been used to measure the same construct.

There are many weaknesses within the current literature, which not only likely account for some of the significant variability on the prevalence of shoulder-related morbidity after neck dissection but also make interpretation of the literature difficult. As can be seen in Table 1, the majority of studies are retrospective and cross-sectional in design with relatively small patient numbers. There is wide variation in inclusion criteria of the different types of neck dissection (ie, selective neck dissection, modified radical neck dissection, and/or radical neck dissection). Furthermore, nonstandardized terminologies have frequently been used in these studies to describe the different types of neck dissections. Some studies included only unilateral neck dissections, whereas others included patients who underwent bilateral neck dissections, although the latter patients may have more impairments and functional limitations than the former. Studies varied in terms of comparative groups; some studies compared outcomes between the different types of neck dissections, whereas others compared preoperative and postoperative differences, or the neck dissection side with the nonsurgical side. Other studies provided no comparison group. There was heterogeneity between studies in terms of the time from surgery when patients were assessed: shortterm $(<6$ months from surgery) versus long-term $(>12$ months) outcomes. Studies that only included patients after 1 year would exclude many of the patients with poor prognosis that do not survive the observation time period, who may have more-advanced disease, and poorer shoulder outcomes. This would potentially bias the results of any study to giving better shoulder outcomes and would also exclude all the patients with temporary morbidity from shoulder paresis.

Another major weakness that needs to be highlighted by this review is the significant variability in patientbased self-report questionnaires used to assess symptoms, activity limitations, and participation restrictions. Despite the fact that the NDII is the only shoulder disability questionnaire that has been specifically developed and validated in patients undergoing neck dissection, ${ }^{41}$ its use has been limited to only a few studies. The lack of a standard questionnaire makes the comparison of results between studies difficult and the literature is compromised by numerous studies that have reported shoulder outcomes using data from questionnaires that were designed by the investigators without consideration of accepted principles of questionnaire development. ${ }^{14,24,35,47,48}$ Although other studies have used questionnaires with acceptable methodologic development, many of these questionnaires were developed for evaluation of other pathologies and diagnoses and have not undergone assessment of their psychometric properties (ie, sensibility, reliability, and validity) in the head and neck patient population. ${ }^{26,28,29,37,40,60,61,67}$

Further, confounding the literature are the various terms that were used by investigators to describe shoulder outcomes after neck dissection, including "shoulder-related quality of life,' "shoulder disability,', "shoulder syndrome,', "shoulder dysfunction,', and "shoulder impairment." This review demonstrates the multiplicity of constructs measured and the variability of the measures that have been used to measure the same construct. Despite using these various terms, few authors clearly described the conceptual framework of the "impairment," "dysfunction," or "disability" they were measuring or reporting. Upon examining each of the studies, it is apparent that the authors were mainly assessing constructs similar to the WHO ICF definition of impairments, activity limitations, and participation restrictions, despite using the term "disability.",

Another drawback to the literature is that the majority of studies seem to focus on symptoms such as pain, impairments in ROM and strength, and limitations in the ability to carry out activities of daily living or recreational activities. There is limited assessment on the impact that impairments and activity limitations have on patients' everyday life (ie, participation restrictions or disability). There have been some reports of changes in occupation status after neck dissection; however, these studies frequently used nonvalidated measures and were mainly assessing occupational status after radical neck dissection and occasionally selective neck dissection. ${ }^{27,52}$ These limitations make evaluation of the literature difficult and need to be recognized in interpreting the review.

Another factor that makes interpretation of the literature difficult is the fact that there is also significant variability between studies in terms of the different treatments patients underwent and the indications. There are differences in terms of whether patients underwent surgery alone versus surgery with either preoperative or postoperative radiotherapy. In addition, patients may have been included that underwent neck dissection for persistent or recurrent disease after radiotherapy. Such differences in interventions may also account for differences in reported outcomes.

Although several authors have tried to assess the impact of the extent of neck dissection on QOL, such an analysis is made difficult by the fact that there are many different factors that contribute to overall QOL in patients with head and neck cancer. Patients with more aggressive neck dissections (ie, radical neck dissection or modified radical neck dissection) often have more advanced disease that in of itself can influence overall scores on global QOL. Furthermore, these same patients may have undergone more extensive resections in addition to adjuvant radiotherapy or chemoradiotherapy that likely will have an even greater impact on a patient's QOL than shoulder-related problems.

In summary, manipulation of cranial nerve XI at the time of neck dissection does result in shoulder pain, reduction in shoulder ROM and strength, and activity limitations related to the shoulder. The extent of cranial 
nerve XI manipulation does influence outcomes. Radical neck dissection is associated with the greatest impairment and activity limitations and selective neck dissection with the least. The extent of shoulder impairment and activity limitations reported in the literature after any type of neck dissection is highly variable. Some of the variability and difficulties in comparing studies is related to the lack of a recognized, uniformly accepted instrument to measure shoulder-related outcomes. Further research is still required, not only to determine the true prevalence of impairments, activity limitations, and participation restrictions, but also to develop and assess ways to predict those at risk for long-term shoulder-related morbidity and to intervene to prevent it from occurring. In order to move forward, a standardized, patient-based self-report measurement tool for assessing shoulder impairments, activity limitations, and participation restrictions, which meets recognized standards for development and evaluation of its psychometric properties in patients with head and neck cancer undergoing neck dissection, needs to be used.

\section{REFERENCES}

1. Hammerlid E, Silander E, Hörnestam L, Sullivan M. Health-related quality of life three years after diagnosis of head and neck cancer - a longitudinal study. Head Neck 2001;23:113-125.

2. Dixon D, Johnston M, McQueen M, Court-Brown C. The Disabilities of the Arm, Shoulder and Hand Questionnaire (DASH) can measure the impairment, activity limitations and participation restriction constructs from the International Classification of Functioning, Disability and Health (ICF). BMC Musculoskelet Disord 2008;9:114.

3. World Health Organization. International Statistical Classification of Diseases and Health Related Problems. 10th Revision, 2nd edition (ICD-10). Geneva Switzerland: World Health Organization Publications; 2004.

4. World Health Organization. International Classification of Functioning, Disability and Health. ICF Geneva, Switzerland: World Health Organization Publications; 2001

5. Ringash J, Bezjak A. A structured review of quality of life instruments for head and neck cancer patients. Head Neck 2001;23:201-213.

6. World Health Organization. WHO expert committee on rehabilitation after cardiovascular diseases, with special emphasis on developing countries. Volume 831 of Technical Reports volume 831 of general history of Africa. Geneva, Switzerland: World Health Organization Publications; 1993.

7. Ewing MR, Martin H. Disability following radical neck dissection; an assessment based on the postoperative evaluation of 100 patients. Cancer 1952:5:873-883.

8. Nahum AM, Mullally W, Marmor L. A syndrome resulting from radical neck dissection. Arch Otolaryngol 1961;74:424-428.

9. Carenfelt C, Eliasson K. Occurrence, duration and prognosis of unexpected accessory nerve paresis in radical neck dissection. Acta Otolaryngol 1980;90:470-473.

10. Szunyogh B. Shoulder disability following radical neck dissection. Am Surg 1959;25:194-198.

11. Bocca E, Pignataro O, Sasaki CT. Functional neck dissection. A description of operative technique. Arch Otolaryngol 1980;106:524-527.

12. Skolnik EM, Tenta LT, Wineinger DM, Tardy ME Jr. Preservation of XI cranial nerve in neck dissections. Laryngoscope 1967;77:1304-1314.

13. Short SO, Kaplan JN, Laramore GE, Cummings CW. Shoulder pain and function after neck dissection with or without preservation of the spinal accessory nerve. Am J Surg 1984;148:478-482.

14. Sobol S, Jensen C, Sawyer W II, Costiloe P, Thong N. Objective comparison of physical dysfunction after neck dissection. Am J Surg 1985;150: 503-509.

15. Hillel A, Patten C. Neck dissection: morbidity and rehabilitation. Cancer Treat Res 1990;52:133-147.

16. Cappiello J, Piazza C, Nicolai P. The spinal accessory nerve in head and neck surgery. Curr Opin Otolaryngol Head Neck Surg 2007;15:107-111.

17. Rogers SN, Ferlito A, Pellitteri PK, Shaha AR, Rinaldo A. Quality of life following neck dissections. Acta Otolaryngol 2004;124:231-236.

18. McGarvey AC, Chiarelli PE, Osmotherly PG, Hoffman GR. Physiotherapy for accessory nerve shoulder dysfunction following neck dissection surgery: a literature review. Head Neck 2011;33:274-280.

19. Shone GR, Yardley MP. An audit into the incidence of handicap after unilateral radical neck dissection. J Laryngol Otol 1991;105:760-762.

20. Dijkstra PU, van Wilgen PC, Buijs RP, et al. Incidence of shoulder pain after neck dissection: a clinical explorative study for risk factors. Head Neck 2001;23:947-953.
21. Fialka V, Vinzenz K. Investigations into shoulder function after radical neck dissection. J Craniomaxillofac Surg 1988;16:143-147.

22. Leipzig B, Suen JY, English JL, Barnes J, Hooper M. Functional evaluation of the spinal accessory nerve after neck dissection. Am J Surg 1983; 146:526-530.

23. Krause HR. Shoulder-arm-syndrome after radical neck dissection: its relation with the innervation of the trapezius muscle. Int J Oral Maxillofac Surg 1992;21:276-279.

24. Saunders JR Jr, Hirata RM, Jaques DA. Considering the spinal accessory nerve in head and neck surgery. Am J Surg 1985;150:491-494.

25. Hillel AD, Kroll H, Dorman J, Medieros J. Radical neck dissection: a subjective and objective evaluation of postoperative disability. J Otolaryngol 1989; 18:53-61.

26. van Wilgen CP, Dijkstra PU, Nauta JM, Vermey A, Roodenburg JL. Shoulder pain and disability in daily life, following supraomohyoid neck dissection: a pilot study. J Craniomaxillofac Surg 2003;31:183-186.

27. Salerno G, Cavaliere M, Foglia A, et al. The 11 th nerve syndrome in functional neck dissection. Laryngoscope 2002;112(7 Pt 1):1299-1307.

28. Güldiken Y, Orhan KS, Demirel T, Ural HI, Yücel EA, Değer K. Assessment of shoulder impairment after functional neck dissection: long term results. Auris Nasus Larynx 2005;32:387-391.

29. Carr SD, Bowyer D, Cox G. Upper limb dysfunction following selective neck dissection: a retrospective questionnaire study. Head Neck 2009;31: 789-792.

30. Oz B, Memis A. Development of musculoskeletal complaints and functional disabilities in patients with laryngeal carcinoma after neck dissection sparing spinal accessory nerve. Eur J Cancer Care (Engl) 2009;18: 179-183.

31. Watkins JP, Williams GB, Mascioli AA, Wan JY, Samant S. Shoulder function in patients undergoing selective neck dissection with or without radiation and chemotherapy. Head Neck 2011;33:615-619.

32. Celik B, Coskun H, Kumas FF, et al. Accessory nerve function after level 2b-preserving selective neck dissection. Head Neck 2009;31:1496-1501.

33. Murer K, Huber GF, Haile SR, Stoeckli SJ. Comparison of morbidity between sentinel node biopsy and elective neck dissection for treatment of the N0 neck in patients with oral squamous cell carcinoma. Head Neck 2011;33:1260-1264

34. Köybasioglu A, Tokcaer AB, Uslu S, Ileri F, Beder L, Ozbilen S. Accessory nerve function after modified radical and lateral neck dissections. $\mathrm{La}$ ryngoscope 2000;110:73-77.

35. El Ghani F, Van Den Brekel MW, De Goede CJ, Kuik J, Leemans CR, Smeele LE. Shoulder function and patient well-being after various types of neck dissections. Clin Otolaryngol Allied Sci 2002;27:403-408.

36. van Wilgen CP, Dijkstra PU, van der Laan BF, Plukker JT, Roodenburg JL. Shoulder complaints after nerve sparing neck dissections. Int J Oral Maxillofac Surg 2004;33:253-257.

37. Chepeha DB, Taylor RJ, Chepeha JC, et al. Functional assessment using Constant's Shoulder Scale after modified radical and selective neck dissection. Head Neck 2002;24:432-436.

38. Erisen L, Basel B, Irdesel J, et al. Shoulder function after accessory nervesparing neck dissections. Head Neck 2004;26:967-971.

39. Rogers SN, Scott B, Lowe D. An evaluation of the shoulder domain of the University of Washington quality of life scale. Br J Oral Maxillofac Surg 2007;45:5-10.

40. Selcuk A, Selcuk B, Bahar S, Dere H. Shoulder function in various types of neck dissection. Role of spinal accessory nerve and cervical plexus preservation. Tumori 2008;94:36-39.

41. Taylor RJ, Chepeha JC, Teknos TN, et al. Development and validation of the neck dissection impairment index: a quality of life measure. Arch Otolaryngol Head Neck Surg 2002;128:44-49.

42. Cheng PT, Hao SP, Lin YH, Yeh AR. Objective comparison of shoulder dysfunction after three neck dissection techniques. Ann Otol Rhinol Laryngol 2000;109(8 Pt 1):761-766.

43. Remmler D, Byers R, Scheetz J, et al. A prospective study of shoulder disability resulting from radical and modified neck dissections. Head Neck Surg 1986;8:280-286.

44. Umeda M, Shigeta T, Takahashi H, et al. Shoulder mobility after spinal accessory nerve-sparing modified radical neck dissection in oral cancer patients. Oral Surg Oral Med Oral Pathol Oral Radiol Endod 2010;109: 820-824.

45. Orhan KS, Demirel T, Baslo B, et al. Spinal accessory nerve function after neck dissections. J Laryngol Otol 2007;121:44-48.

46. Zibordi F, Baiocco F, Bascelli C, Bini A, Canepa A. Spinal accessory nerve function following neck dissection. Ann Otol Rhinol Laryngol 1988; 97:83-86.

47. Shah S, Har-El G, Rosenfeld RM. Short-term and long-term quality of life after neck dissection. Head Neck 2001;23:954-961.

48. Cappiello J, Piazza C, Giudice M, De Maria G, Nicolai P. Shoulder disability after different selective neck dissections (levels II-IV versus levels II-V): a comparative study. Laryngoscope 2005;115:259-263.

49. Tsuji T, Tanuma A, Onitsuka T, et al. Electromyographic findings after different selective neck dissections. Laryngoscope 2007;117:319-322.

50. Inoue H, Nibu K, Saito M, et al. Quality of life after neck dissection. Arch Otolaryngol Head Neck Surg 2006;132:662-666. 
51. Nibu K, Ebihara Y, Ebihara M, et al. Quality of life after neck dissection: a multicenter longitudinal study by the Japanese Clinical Study Group on Standardization of Treatment for Lymph Node Metastasis of Head and Neck Cancer. Int J Clin Oncol 2010;15:33-38.

52. Schuller DE, Reiches NA, Hamaker RC, et al. Analysis of disability resulting from treatment including radical neck dissection or modified neck dissection. Head Neck Surg 1983;6:551-558.

53. Kuntz AL, Weymuller EA Jr. Impact of neck dissection on quality of life. Laryngoscope 1999;109:1334-1338.

54. Terrell JE, Welsh DE, Bradford CR, et al. Pain, quality of life, and spinal accessory nerve status after neck dissection. Laryngoscope 2000;110: 620-626.

55. Laverick S, Lowe D, Brown JS, Vaughan ED, Rogers SN. The impact of neck dissection on health-related quality of life. Arch Otolaryngol Head Neck Surg 2004;130:149-154.

56. Schiefke F, Akdemir M, Weber A, Akdemir D, Singer S, Frerich B. Function, postoperative morbidity, and quality of life after cervical sentinel node biopsy and after selective neck dissection. Head Neck 2009;31: 503-512.

57. King PS, Lewis FR, Weddle JL, Fowlks EW. Effect of radical neck dissection on total rehabilitation of the laryngectomee. Am J Phys Med 1973;52: $1-17$.

58. Hillel AD. Disability resulting from radical and modified neck dissections. Head Neck Surg 1986;9:127-129.
59. van Wilgen CP, Dijkstra PU, van der Laan BF, Plukker JT, Roodenburg JL. Shoulder complaints after neck dissection; is the spinal accessory nerve involved? Br J Oral Maxillofac Surg 2003;41:7-11.

60. Stuiver MM, van Wilgen CP, de Boer EM, et al. Impact of shoulder complaints after neck dissection on shoulder disability and quality of life. Otolaryngol Head Neck Surg 2008;139:32-39.

61. Koybaşioğlu A, Bora Tokçaer A, Inal E, Uslu S, Koçak T, Ural A. Accessory nerve function in lateral selective neck dissection with undissected level IIb. ORL J Otorhinolaryngol Relat Spec 2006;68:88-92.

62. Flores AJ, Lavernia CJ, Owens PW. Anatomy and physiology of peripheral nerve injury and repair. Am J Orthop (Belle Mead NJ) 2000;29: $167-173$.

63. van Wilgen CP, Dijkstra PU, van der Laan BF, Plukker JT, Roodenburg JL. Shoulder and neck morbidity in quality of life after surgery for head and neck cancer. Head Neck 2004;26:839-844.

64. Krause HR, Bremerich A, Herrmann M. The innervation of the trapezius muscle in connection with radical neck-dissection. An anatomical study. J Craniomaxillofac Surg 1991;19:87-89.

65. Patten C, Hillel A. The 11 th nerve syndrome. Accessory nerve palsy or adhesive capsulitis? Arch Otolaryngol Head Neck Surg 1993;119:215-220.

66. Soo KC, Strong EW, Spiro RH, Shah JP, Nori S, Green RF. Innervation of the trapezius muscle by the intra-operative measurement of motor action potentials. Head Neck 1993;15:216-221.

67. van Wilgen CP, Dijkstra PU, van der Laan BF, Plukker JT, Roodenburg JL. Morbidity of the neck after head and neck cancer therapy. Head Neck 2004;26:785-791. 